\title{
INTERIORIZAÇÃO DA AIDS EM MUNICÍPIO DE MÉDIO PORTE DO RIO GRANDE DO SUL (2008-2012): aspectos importantes para políticas de saúde
}

\author{
INTERNALIZATION OF AIDS IN A MEDIUM MUNICIPALITY \\ IN RIO GRANDE DO SUL (2008-2012): \\ important aspects for health policies
}

\section{Édina Stadler Schossler', Guilherme Adam Fraga², João Guilherme Stadler Schossler Adalvane Nobres Damaceno ${ }^{4}$, Sandra Trevisan Beck ${ }^{5}$, Teresinha Heick Weiller ${ }^{6}$}

\section{RESUMO}

A epidemia mundial da AIDS, apesar de concentrada principalmente em grandes centros, tem se disseminado para municípios de médio e pequeno porte. Desse modo, analisaram-se critérios relativos à epidemia entre o município de Santa Maria, o Estado do Rio Grande do Sul e o Brasil, entre 2008 e 2012, bem como as regiões administrativas municipais de maior taxa de detecção da doença em 2010, com o objetivo de comparar o perfil da doença no município com o perfil nacional. Foram utilizadas informações provenientes dos bancos de dados do Sistema Nacional de Agravos Notificáveis e Instituto Brasileiro de Geografia e Estatística , constituindo-se em um estudo descritivo, quantitativo. $O$ perfil encontrado foi semelhante ao nacional, predominando a transmissão por relação sexual, com a doença acometendo principalmente indivíduos adulto-jovens, da raça branca e com baixa escolaridade. Desta forma evidencia-se a necessidade de políticas públicas de saúde com foco especial nesta população.

Descritores: Síndrome da Imunodeficiência Adquirida; Epidemiologia; Promoção da Saúde.

\section{ABSTRACT}

The worldwide AIDS epidemic, despite being concentrated in large centers, has spread to small and medium municipalities. In this way we analyzed epidemic criteria among a southern Brazil city, the state of Rio Grande do Sul and Brazil, between 2008 and 2012, as well as the municipal administrative regions whose detection rate of the disease was the highest in 2010 , in order to compare the national and the local profile of the disease. We have worked with information from Sistema Nacional de Agravos Notificáveis and from Instituto Brasileiro de Geografia e Estatística database, what makes it a quantitative a descriptive study. The local profile was similar to the national profile, with predominant transmission by sexual intercourse, with the disease affecting mainly young adult individuals, white race and low education. Thus, public health policies should have a special focus on this population.

Descriptors: Acquired Immune Deficiency Syndrome; Epidemiology; Health Promotion.
${ }^{1}$ Graduanda em Medicina na Universidade Federal de Santa Maria (UFSM), Santa Maria, RS, Brasil.

${ }^{2}$ Graduando em Medicina na Universidade Federal de Santa Maria (UFSM), Santa Maria, RS, Brasil.

${ }^{3}$ Graduado em Medicina pela Universidade Federal de Santa Maria (UFSM), Santa Maria, RS, Brasil.

\section{${ }^{4}$ Mestre em Enfermagem pela Universidade Federal de Santa Maria (UFSM), Santa Maria, RS, Brasil.}

${ }^{5}$ Doutora Farmacêutica pela Universidade de São Paulo (USP), São Paulo, SP, Brasil.

${ }^{6}$ Doutora em Enfermagem em Saúde Pública pela Universidade de São Paulo (USP), São Paulo, SP, Brasil. 


\section{Introdução}

Entendendo território como as dimensões econômicas, políticas, culturais e epidemiológicas, configura-se nesse espaço social uma determinada realidade de saúde da população que nele vive, a qual se encontra em permanente movimento, por isso denominada de processo saúde-doença ${ }^{1}$. Neste contexto, para a interpretação do processo saúde-doença, considera-se que o risco indica probabilidades e a vulnerabilidade é um indicador da iniquidade e da desigualdade social. Nesta perspectiva, segundo Ayres ${ }^{2}$, a vulnerabilidade expressa os potenciais de adoecimento, de não adoecimento e de enfrentamento relacionados a todo e a cada indivíduo. Dessa forma, a exposição a agravos de saúde resulta de aspectos individuais e de contextos ou condições coletivas que produzem maior suscetibilidade aos agravos e morte e, simultaneamente, à possibilidade e aos recursos para o seu enfrentamento.

Sabe-se que o conhecimento sobre a distribuição das necessidades de saúde é fundamental para a implantação de uma política de saúde equitativa. Nesse sentido, cabe destacar que o princípio da equidade pressupõe que as ações de saúde levem em consideração a distribuição heterogênea das necessidades da população e as diferentes formas de hierarquização das prioridades para intervenção ${ }^{3}$.

Segundo dados da Organização das Nações Unidas (ONU), a epidemia da AIDS está concentrada nos grandes centros urbanos, onde também estão as maiores proporções de casos de AIDS nas populações em situação de maior vulnerabilidade sendo a quinta principal causa de morte entre adultos e a principal causa de morte entre mulheres na faixa etária de 15 a 49 anos. No entanto, esta epidemia tem se disseminado dos grandes centros para municípios de médio e pequeno porte ${ }^{4}$.

No Brasil, estima-se que aproximadamente 734 mil pessoas vivam com o Vírus da Imunodeficiência Humana (HIV)/ AIDS, o que representa uma taxa de prevalência de $0,4 \%$ na população geral, com razão segundo o sexo masculino/feminino de 1,8 em relação a taxa de detecção de AIDS $^{5}$. A epidemia brasileira nas populações em situação de vulnerabilidade, no período de 2009 a 2013 apresentaram uma prevalência de 11,7\% entre homens que fazem sexo com homens (HSH), 4,9\% entre profissionais do sexo e entre usuários de drogas 5,9\%. Em 2013, a taxa de detecção de AIDS entre as Unidades da Federação indica que os estados do Rio Grande do Sul e Amazonas apresentam as maiores taxas, com valores de 41,3 e 37,4 casos para cada 100 mil habitantes. Porto Alegre é a capital com a maior taxa registrada em 2013, mais que o dobro da taxa do estado e quase cinco vezes a taxa do Brasil (96,2 casos para cada 100 mil habitantes ${ }^{5}$ ).

Estes dados mostram a necessidade de ações efetivas para o controle da infecção. O Pacto pela saúde tem como objetivo eleger prioridades nacionais e incentivar que estados e municípios elejam as suas a partir da realidade local e regional. Este Pacto possui três dimensões: Pacto pela Vida, Pacto em Defesa do SUS e Pacto de Gestão, que reforça a regionalização, a territorialização da saúde como base para a organização dos sistemas, estruturando as regiões sanitárias. O Pacto pela Vida é o compromisso entre os gestores do SUS em torno de prioridades que apresentam impacto sobre a situação de saúde da população brasileira. A definição de prioridades deve ser estabelecida por meio de metas nacionais, estaduais, regionais ou municipais. Prioridades estaduais ou regionais podem ser agregadas às prioridades nacionais, conforme pactuação local. São seis as prioridades pactuadas: Saúde do Idoso; Controle do câncer do colo do útero e da mama; Redução da mortalidade infantil e materna; Fortalecimento da capacidade de resposta às doenças emergentes e endemias, Promoção da Saúde e Fortalecimento da Atenção Básica ${ }^{6}$.

O presente estudo, realizado com a participação de alunos de graduação, integrantes do Programa de Educação pelo Trabalho para a Saúde (PET-Saúde) junto à Universidade Federal de Santa Maria, vem no sentido de responder uma necessidade/prioridade da vigilância em saúde no município, visando identificar as áreas de vulnerabilidade de doenças de notificação compulsória (DNC) relacionadas no pacto pela vida, enfatizando a Síndrome da Imunodeficiência Adquirida (AIDS) uma vez que Santa Maria encontra-se entre os municípios brasileiros com mais de 100 mil habitantes em $10^{\circ}$ lugar no que se refere ao numero de casos notificados ${ }^{5}$. Assim, este estudo teve como objetivo analisar e comparar o perfil dos casos de AIDS em maiores de 13 anos no país, no estado do Rio Grande do Sul e município de Santa Maria/RS.

\section{Metodologia}

Foi realizado um estudo descritivo, quantitativo, de dados secundários, no qual foram incluídos e analisados, os casos de AIDS em maiores de treze anos registrados no município de Santa Maria/RS no período de 2008 a 2012, comparando a situação desse município com a do estado do Rio Grande do Sul e do Brasil. 
A cidade de Santa Maria é uma das principais cidades do interior do Rio Grande do Sul, podendo ser referencia para o estudo da infecção pelo HIVIAIDS em menores de 13 anos no interior do estado. A cidade está localizada na região Central do Rio Grande do Sul, e possui uma população de 261.031 habitantes fixos ${ }^{7}$, incluída a zona urbana e rural, e mais uma população flutuante de 30 mil pessoas formada, principalmente, por estudantes e militares de onze quartéis do Exército e uma unidade da Aeronáutica, constituindo o segundo maior centro militar do Brasil.

Os dados foram obtidos através da análise dos casos notificados de AIDS em maiores de 13 anos do município de Santa Maria, incluídas no banco do Sistema de Informação de Agravos de Notificação (SINAN-net), no período de janeiro de 2008 a dezembro de 2012, totalizando 690 casos de AIDS. Os dados foram coletados entre os meses de junho a setembro de 2013. Foram excluídos os casos notificados em indivíduos menores de treze anos.

Para a demonstração inicial da situação epidemiológica da AIDS no município de Santa Maria, foram utilizados dados de 2001 a 2012, como forma de melhor visualização da prevalência em longo prazo.

Para análise do perfil dos indivíduos notificados com AIDS no município $(n=690)$, foi utilizado para estudo um período de cinco anos (2008-2012), e para determinar as regiões administrativas com maior taxa de incidência de caso de AIDS notificados no município, foi utilizado o ano de 2010, por ser o ano com maior número de notificações de casos de AIDS no período de estudo. As taxas de incidência foram calculadas baseadas na população estimada pelo censo do Instituto Brasileiro de Geografia e Estatística (IBGE) do ano de $2010^{7}$ A partir deste dado, considerando as oito regiões administrativas, excluindo a área rural, foram analisados os seguintes fatores: sexo, raça, modo de aquisição, escolaridade e faixa etária.

Quanto aos critérios utilizados para definir o modo de contágio do vírus HIV quando o indivíduo tinha mais de um fator de risco para a contaminação, foram definidos como prioridade de modo de aquisição, respectivamente, transmissão vertical, o uso de drogas, transfusão sanguínea e, por último, a exposição sexual.

Os dados foram analisados por meio de análise estatística descritiva, utilizando-se o programa SPSS (Statistical Package for the Social Sciences) versão 13.0.

O estudo fez parte do trabalho de equipe pertencente ao programa PRO-PET Saúde, desenvolvido na Universidade Federal de Santa Maria com o apoio do Ministério da Saúde, e faz parte de um projeto aprovado pelo CEP-UFSM registro CAAE: 02250.0.243.000-11.

\section{Resultados}

\section{Situação do município}

Conforme a série histórica, considerando o número de casos de AIDS notificados entre 2001 e 2012, verifica-se um aumento com tendência linear na taxa de incidência, apresentando maior variação nos últimos cinco anos do período estudado, refletindo o aumento do número de indivíduos infectados pelo vírus HIV no município (figura 1).

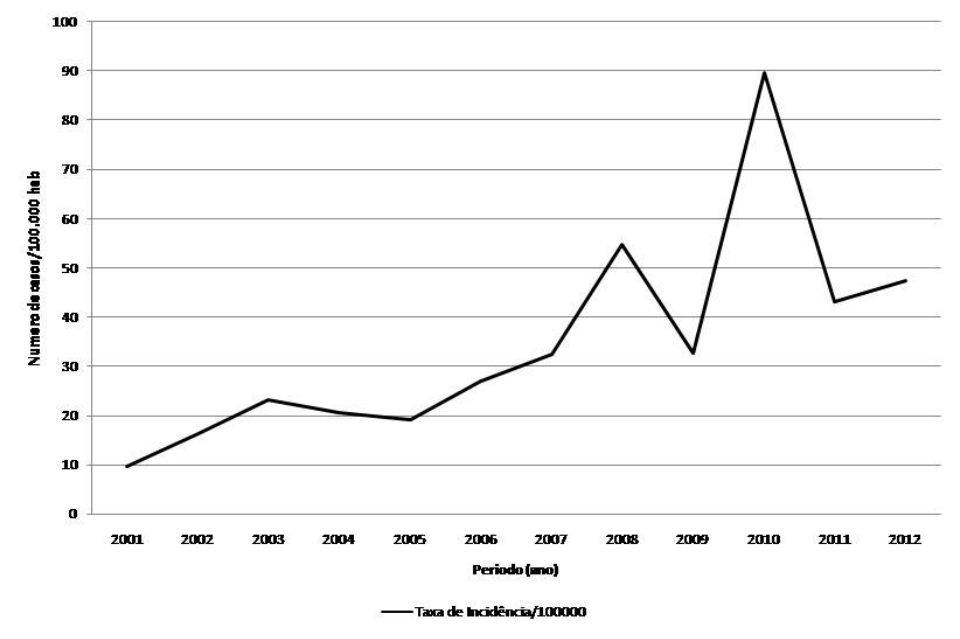

Figura 1. Casos notificados de AIDS em indivíduos maiores de treze anos no município de Santa Maria - RS (Série Histórica 2001-2012) 
No período entre 2008 a 2012, analisando a distribuição do número de casos notificados, segundo a região administrativa, é possível verificar que as regiões oeste e centro-urbano, que são as mais populosas do município, concentram grande número de casos (figura 2).

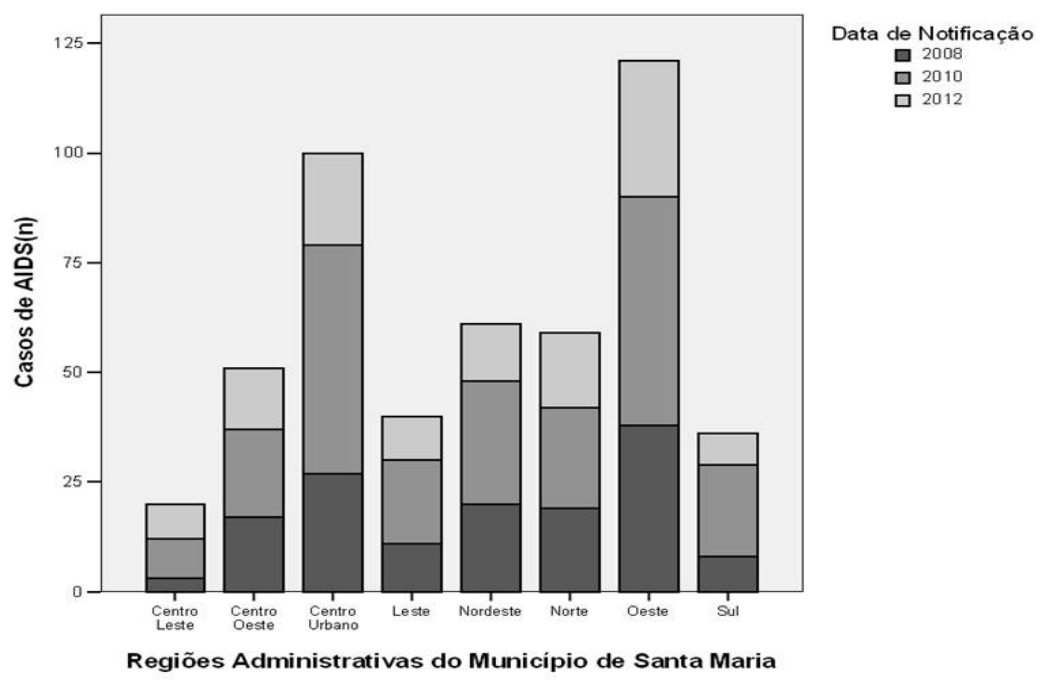

Figura 2. Número de casos de AIDS (2008-2010-2012) por região administrativa do Município de Santa Maria- RS

Do total de casos analisados ( $n=690), 75,8 \%$ corresponderam à raça branca e $10,7 \%$ à raça preta, com discreto predomínio do sexo masculino (58,6\%). A faixa etária mais acometida pela epidemia é a dos 30 aos 34 anos, com uma percentagem levemente maior $(20,2 \%)$ que a faixa etária entre 40 a 49 anos (18,8\%). O menor segmento notificado é de indivíduos com mais de 60 anos, correspondendo a 3,3\% (23/690) dos casos. Em relação à categoria de exposição à infecção, a forma sexual predominou em todas as faixas etárias e em todas as raças, exceto nos indígenas. $O$ único caso de AIDS notificado nessa raça foi devido ao uso de drogas.

Ao ser relacionado o item raça com sexo ou modo de aquisição, percebe-se que entre os indivíduos de raça branca houve o predomínio do sexo masculino (60\%). Já quando analisados os indivíduos de raça preta, houve o predomínio do sexo feminino $(58,1 \%)$ sobre o masculino. Nos pardos, a maioria era do sexo masculino $(61,1 \%)$.

Entre os indivíduos notificados com AIDS entre 2008 a 2012 no município, não considerando a perda de $27,7 \%$ de casos com informação ignorada e 7,4\% de perdas em relação à escolaridade, houve predomínio da transmissão sexual, em todas as raças, entre indivíduos com escolaridade da $5^{\mathrm{a}}$ a $8^{\circ}$ série incompleta em todas as regiões (68,2\%), exceto na região administrativa Centro Oeste, na qual predominou a transmissão por uso de droga $(55,56 \%)$. Ressalta-se que entre os indivíduos com ensino superior completo, $71,43 \%$ são residentes na região administrativa Centro Urbano, sendo que, entre eles, há predomínio da transmissão sexual (95\%).

Analisando os indivíduos que adquiriram a infecção através de relação sexual, verifica-se que a faixa etária prevalente situa-se entre 40 a 49 anos (20,1\%), seguida dos indivíduos entre 30 a 34 anos (18,1\%) e 25 a 29 anos (16\%). Nos indivíduos com mais de 60 anos, a relação sexual foi a única forma de transmissão notificada entre todos os indivíduos desse grupo no período. Dentre os casos de transmissão sexual, destacam-se a relação heterossexual, que perfazem um total de 76,6\%.

Já entre os indivíduos que adquiriram a infecção através do uso de drogas, predominou a faixa etária dos 30 a 34 anos de idade, com $24,7 \%$ do total de casos notificados.

Ao ser analisada a forma de aquisição da infecção, dentro de cada faixa etária, verifica-se que a transmissão por uso de drogas foi relevante entre indivíduos que se encontravam na faixa etária entre 13 a 19 anos (40\% dos casos). Nas demais faixas etárias, a frequência da transmissão por uso de drogas foi: 24,1\% (20 a 24 anos), 23,6\% (25 a 29 anos), 28,4\% (30 a 34 anos), 29,9\% (35 a 39 anos), 17,7\% (40 a 49 anos) e 7,7\% (50 a 59 anos), não havendo casos de transmissão por uso de droga em maiores de 60 anos. 


\section{Situação nas regiões administrativas do município com maior taxa de detecção de casos de HIV/AIDS}

Partindo do ano com maior número de notificações de casos de AIDS no município (2010), o cálculo da taxa de incidência de casos notificados para cada uma das oito regiões administrativas mostrou que as regiões Nordeste e Sul foram as que apresentaram a maior incidência, embora alguns bairros que pertençam a estas regiões se caracterizem por não apresentar notificação de AIDS no período (figura 3).

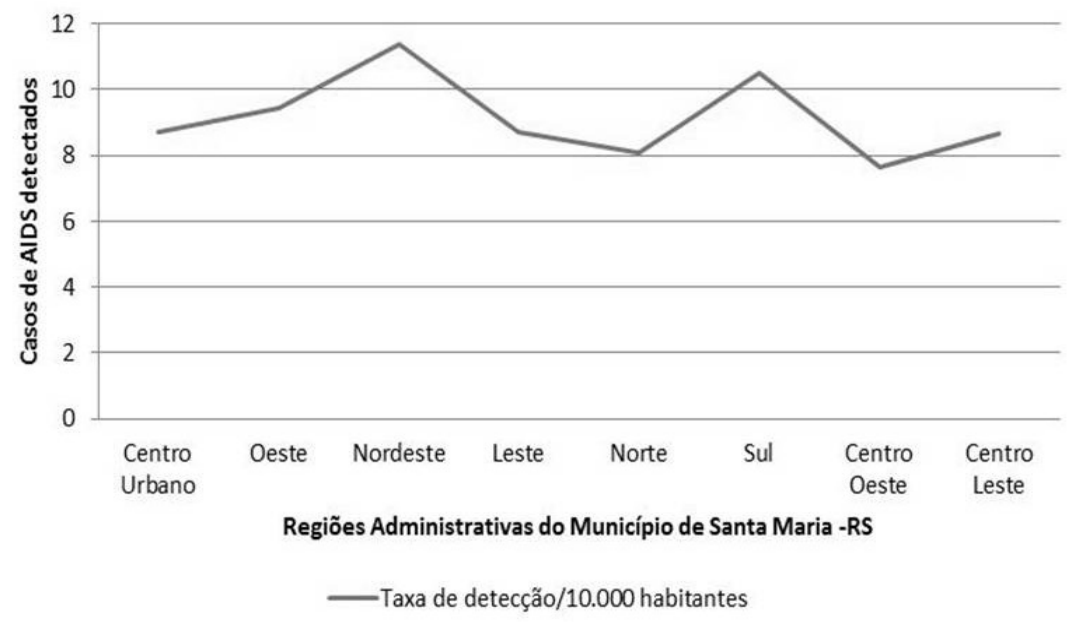

Figura 3. Taxa de detecção de AIDS em maiores de 13 anos nas diferentes regiões administrativas do município de Santa Maria/RS no ano de 2010.

O perfil dos indivíduos notificados com AIDS no período entre 2008 a 2010, residentes nas duas regiões administrativas identificadas com maior taxa de incidência de AIDS (nordeste e sul) quando considerada escolaridade, mostrou que $27,3 \%$ dos indivíduos possuíam instrução da $5^{\text {a }}$ a $8^{\text {a }}$ serie incompleta do ensino fundamental, $12,2 \%$ possuíam ensino médio completo e 10,8\% ensino fundamental completo. Ressalta-se que, nesse critério, o item ignorado correspondeu a $18 \%$ e as perdas totalizaram $8,6 \%$. A faixa etária predominante, com $25,9 \%$ dos casos, foi de indivíduos com idade entre 30 a 34 anos. A maior parte da população é branca $(74,1 \%)$ e do sexo masculino (59\%).

Com relação à forma de aquisição, na totalidade dos indivíduos das duas regiões, a transfusão sanguínea e a transmissão vertical aparecem de forma discreta, com menos de $5 \%$ dos casos. 0 uso de drogas injetáveis foi relatado em $29 \%$ dos casos, sendo a transmissão sexual $(66,2 \%)$ a forma mais frequente da contaminação pelo HIV.

O predomínio de transmissão por uso de drogas também prevaleceu apenas na faixa etária dos 35 a 39 anos, com $57,1 \%$ dos casos, enquanto na faixa etária dos 13 aos 19 anos a transmissão por uso de drogas foi igual a sexual, sendo $50 \%$ cada uma. Entre os indivíduos com ensino superior completo destas regiões, a transmissão foi exclusivamente sexual. 0 comportamento referente à orientação sexual mostra que a relação heterossexual é a mais frequente, com 69,1\% dos casos (figura 4). 


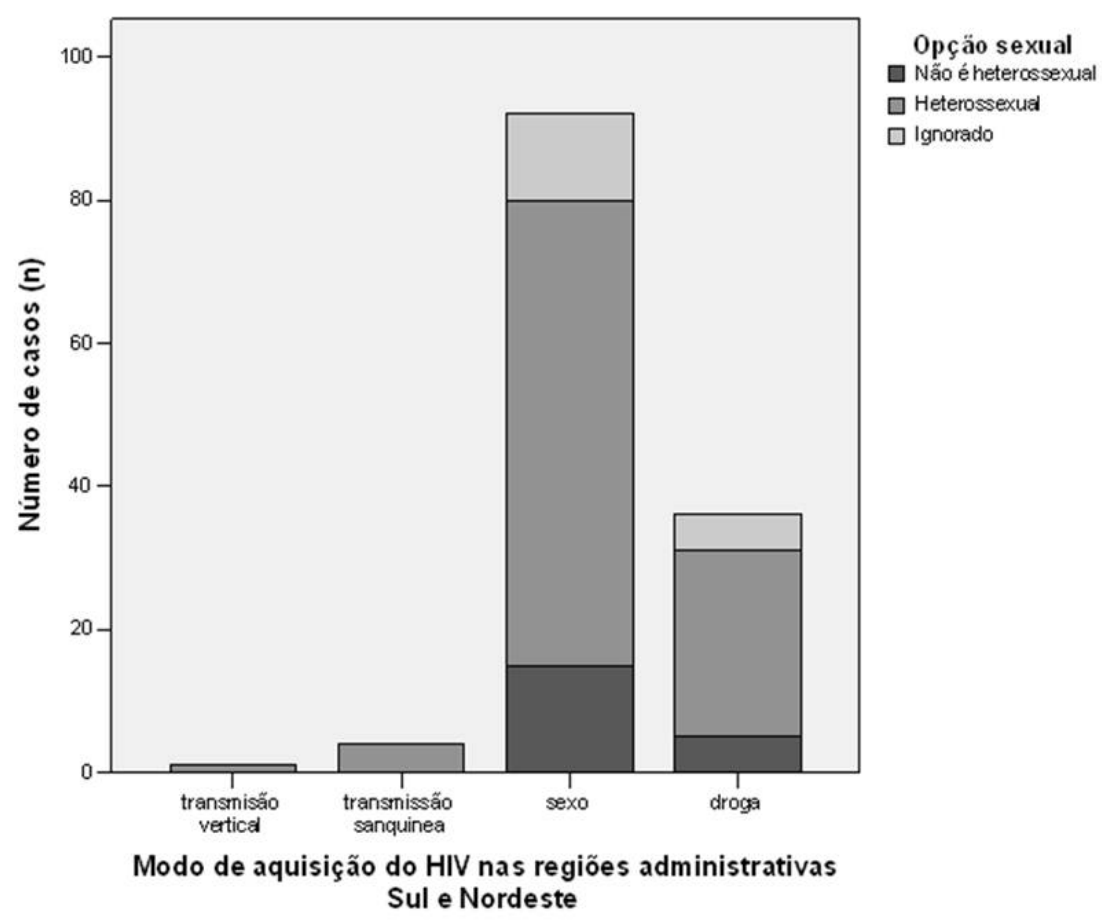

Figura 4: Modo de aquisição e orientação sexual dos indivíduos residentes nas duas regiões administrativas com maior detecção de casos de AIDS em 2010.

Os resultados apresentados apresentam limitações que correspondem àquelas encontradas em pesquisas que utilizam dados secundários, que podem comprometer a validade de seus resultados e conclusões, isso porque existe alto índice de falhas no preenchimento das fichas de notificação, resultando em dados incompletos, rotineiramente identificados como ignorados, demonstrando a má qualidade do processo de notificação. Além disso, há subnotificação de casos no SINAN, em virtude de o número de registros "sem informação" ser alto, resultando em elevado número de perdas. Ademais, é notória a existência de indivíduos com AIDS não registrados no SINAN.

Soma-se ao já exposto, o fato de a população do censo do IBGE, referente ao ano de 2010, utilizada pelo estudo, não excluir os indivíduos com idade inferior a treze anos, o que determina uma possível variação na incidência calculada, uma vez que os casos de AIDS estudados correspondem exclusivamente a indivíduos maiores de treze anos.

\section{Discussão}

A alta prevalência de AIDS em Santa Maria, município do interior do Rio Grande do Sul, ilustra o já conhecido conceito de interiorização da doença, demonstrado por diversos estudos realizados nos últimos anos em outras regiões do país $8,9,10,11$. A população afetada pela epidemia de AIDS em Santa Maria apresentou características semelhantes ao Brasil, sendo predominantemente do sexo masculino, branca, heterossexual e adulta-jovem.

A análise do perfil das regiões com maior detecção de casos de AIDS não difere do encontrado na população geral do município de Santa Maria. Isso se deve, possivelmente, à semelhança entre o perfil das duas regiões e o de todo o município. Nesse contexto, levantou-se a possibilidade de a pauperização ser um fator comum a esses indivíduos, como já demonstrado em estudos anteriores ${ }^{9,12,13}$, ainda que não se possa afirmar a existência de relação plena entre renda e escolaridade. Um fator limitante foi a impossibilidade de relacionar a pauperização ao nível socioeconômico por região administrativa do município de Santa Maria, uma vez que estes dados não se encontram disponíveis segregados por região administrativa.

Nota-se que a faixa etária mais acometida pela epidemia é aquela dos 30 aos 34 anos, com uma percentagem levemente maior $(20,2 \%)$ que na faixa entre 40 a 49 anos $(18,8 \%)$, corroborando a tendência de aumento da idade média do indivíduo acometido, constatada desde $1998^{10}$. Esses dados estão de acordo com estudos de base nacional e internacional para estimativa do perfil da doença ${ }^{14,15,16,17}$. 
Além disso, o perfil do indivíduo com AIDS no Brasil é marcado pela prevalência do sexo masculino. Já em relação à raça, foi observado aumento da taxa de detecção da doença entre os de cor preta nos últimos dez anos em ambos os sexos. Apesar disto, observa-se uma tendência de redução no numero de casos entre este grupo e de aumento entre os brancos e pardos 5 .

No presente estudo, observamos que entre os indivíduos de raça branca houve o predomínio do sexo masculino, e entre os de raça preta, do sexo feminino. Essa situação também foi observada por outros pesquisadores. Em estudo de $2013^{18}$, concluiu-se que a discriminação racial influencia o desenvolvimento da autoestima e contribui para a construção de uma identidade negativa, que, aliada ao racismo e à pobreza, configura-se num contexto de vulnerabilidade às DST/ AIDS, considerando-se, também, que mulheres negras estão nos mais baixos patamares de renda ${ }^{19}$. Nesse mesmo contexto, é sabido que adolescentes negras são as vítimas preferenciais de abuso sexual e de exploração sexual comercial ${ }^{20}$. Além disso, mulheres negras apresentam risco aumentado de infecções genitais e estão numa posição de menor poder de negociação de práticas sexuais seguras, em consequência da desigualdade de gênero, além de não terem reconhecida a legitimidade de seu exercício sexual ${ }^{21}$.

Do mesmo modo, nacionalmente, em relação à idade, ocorre o predomínio dos 30 a 49 anos, enquanto o modo de aquisição é principalmente através do sexo e a escolaridade predomina da $5^{\circ}$ a $8^{\circ}$ série incompleta ${ }^{5}$. Tais achados não diferem do encontrado no município de Santa Maria a partir análise dos casos de AIDS notificados no período de 2008 a 2012, em todas as regiões administrativas.

A transmissão sexual foi a principal forma de aquisição do vírus, o que também vai ao encontro do demostrado por outros estudos $22,23,24$, com predominância das relações heterossexuais como forma de transmissão. É válido ressaltar que a única forma de transmissão em idosos foi a sexual. Isso evidencia a importância de medidas de prevenção que contemplem a sexualidade na terceira idade, uma vez que os idosos devem ser vistos como indivíduos que possuem desejos e necessidades sexuais ${ }^{25}$. A baixa percepção de vulnerabilidade pelos idosos aumenta o risco de infecção nessa população, concepção muitas vezes disseminada pelos próprios profissionais da saúde, sendo que, ao desconsiderar os mais velhos como sexualmente ativos, perdem a oportunidade do diagnóstico precoce ${ }^{26}$.

O modo de transmissão sexual predominou em todas as raças, exceto nos indígenas, em que foi relatado um único caso, cuja transmissão ocorreu através do uso de drogas. Existem poucos estudos preocupados em relatar a AIDS em indígenas. As doenças sexualmente transmissíveis, incluindo a infecção pelo HIV em população indígena são pouco conhecidas e notificadas no Brasili ${ }^{27}$. Autores que abordaram a temática ${ }^{28}$ defendem que 0 crescimento das cidades elevou o contingente populacional em regiões próximas de áreas indígenas, provocando uma aproximação forçada entre os índios e a população não indígena. Isso trouxe para as tribos indígenas problemas previamente desconhecidos, como álcool, drogas e AIDS.

É importante observar que a transmissão pelo uso de drogas não aparece como forma relevante. Estudo de fatores de risco para transmissão do HIV em usuários de drogas mostrou que existe um maior risco de infecção neste grupo para indivíduos com mais de trinta anos, renda familiar de menos de um salário mínimo, e com menos de sete anos de escolaridade ${ }^{15,29,30,31}$ semelhante ao encontrado no presente estudo. Contudo, os dados aqui apresentados mostram que entre indivíduos mais jovens, na faixa etária entre 13 a 19 anos, em $50 \%$ dos casos a forma de transmissão foi por uso de drogas. Este fato ressalta a importância da instituição de medidas de prevenção dessa epidemia, nessa população. Nesse sentido, as estratégias de educação em saúde voltam-se, majoritariamente, à prevenção da AIDS através do estimulo ao uso de preservativos, subestimando o alto potencial de transmissão do vírus HIV pelo uso de drogas.

A variável "anos de estudo" indicou um nível de instrução concentrado na categoria fundamental incompleto, embora Santa Maria também apresente índices relevantes nas categorias "fundamental completo" e "ignorado", caracterizando essa população como de baixo grau de escolaridade e menor qualificação profissional. Estas características expressam a preocupação, já evidenciada em estudos anteriores, em demarcar uma transição no perfil epidemiológico que limitava a AIDS aos principais centros urbanos e a determinados grupos de risco nos anos 1980, 12,13,32.

O presente estudo mostra a feminização da epidemia, já evidenciada ao longo dos anos. Essa feminização denota associação com a forma de transmissão heterossexual e com o aumento do risco de transmissão vertical do vírus HIV ${ }^{14,33}$. $O$ aumento de casos de AIDS em mulheres, associado à maior ocorrência durante a idade fértil, é sinal de alerta ao sistema de saúde e indicativo para o fortalecimento de medidas de promoção da saúde em relação à transmissão vertical ${ }^{17}$.

Quanto à disseminação da epidemia pelas áreas estudadas, têm-se duas principais formas prováveis de propagação: a difusão por meio de contiguidade e a difusão hierárquica por redes ${ }^{34}$. A difusão por meio de contiguidade ocorre pelo contato entre áreas adjacentes devido à frequência de interações entre os habitantes, enquanto a difusão por redes permite a transmissão dentro de comunidades, entre pessoas participantes de redes sociais, mesmo que 
distantes. Uma vez que as maiores incidências da epidemia encontram-se nas regiões nordeste e sul do município, portanto afastadas geograficamente, constata-se que a difusão por redes é a que melhor explica as altas taxas de casos notificados nessas regiões. Deve-se ressaltar, porém, que o processo de difusão não é homogêneo e produz uma mudança no perfil da epidemia à medida que esta alcança novas populações ${ }^{35}$.

Por fim, o desconhecimento do perfil dos pacientes com AIDS gera uma gestão de saúde ineficiente. Dessa forma, este estudo, assim como outros que abordem o perfil da doença na sua comunidade, contribui para o adequado planejamento de ações em saúde no âmbito da AIDS, enriquecendo os dados disponíveis aos gestores para 0 desenvolvimento de políticas públicas de saúde que realmente abordem a população necessitada.

As políticas públicas de saúde relacionadas ao HIVIAIDS devem visar à saúde integral do paciente, tendo como foco o desenvolvimento de uma linha de cuidado, que atenda as necessidades deste indivíduo desde a porta de entrada até 0 atendimento de alta complexidade. Neste aspecto, os Centros de Testagem e Aconselhamento (CTA), têm o papel de promover a identificação precoce de pacientes HIV positivo para o imediato encaminhamento à rede de assistência. Os CTA garantem o anonimato flexível e, através de resultados garantem o encaminhamento dos indivíduos HIV positivos para serviços de referência assistencial, onde poderão ter acesso ao tratamento ${ }^{36}$.

Não menos importante é a necessidade de ações que qualifiquem os profissionais envolvidos na rede de atenção a saúde do usuário. Promover ações qualificadas com foco no comportamento do indivíduo (como uso de preservativo, debates sobre formas de prevenção, ampliação do acesso ao diagnóstico), que atinjam diferentes populações, como população idosa, homens heterossexuais, população indígena, com atenção a populações mais vulneráveis como usuários de drogas, homossexuais, profissionais do sexo, poderá contribuir para a prevenção e transmissão da infecção.

\section{Considerações Finais}

Os resultados aqui apresentados revelaram um perfil da doença semelhante ao encontrado em dados nacionais e estaduais, mostrando o predomínio da AIDS em indivíduos adulto-jovens, heterossexuais, brancos, com baixa escolaridade, sendo a transmissão principalmente via sexual.

Apesar das limitações identificadas, este estudo acrescentou informações importantes acerca da situação epidemiológica da AIDS em um município de porte médio, identificando áreas com maior vulnerabilidade, onde as ações de saúde deverão ser intensificadas. Tendo como enfoque a educação em saúde, contribuiu para a formação de futuros profissionais, de diferentes áreas acadêmicas, que serão multiplicadores do conhecimento, mostrando a importância da correta notificação dos casos de AIDS, para o planejamento eficiente das ações de saúde.

\section{Referências}

1. Takeda S. A organização de Serviços de Atenção Primária à Saúde. In: Duncan, Bruce B.e colaboradores. Medicina Ambulatorial: condutas de Atenção Primária Baseadas em Evidências. Porto Alegre: Artmed; 2004.p 05-13.

2. Ayres JRCM, Paiva V, Franca I, Gravato N, Lacerda R, Negra MD, et al. Vulnerability, HumanRights, And Comprehensive Health Care Needs Of Young People Living With HIVIAIDS. Am J Public Health. 2006; 96(6): 1001-1006.

3. Ministério da Saúde. Secretaria de Atenção à Saúde. Departamento de Atenção Básica. Monitoramento na Atenção Básica de saúde: roteiros para reflexão e ação. $1^{\text {a }}$ ed Brasília DF: Editora MS; 2004.

4. Organização Mundial da Saúde. A ONU e a resposta à AIDS no Brasil. 2013. [Internet] [acesso em Jun 2014]. Disponível em: http://www.unaids.org.br/documentos/A\%200NU\%20e\%20a\%20resposta\%20-\%20PORTUGU\%C3\%8AS.pdf .

5. Ministério da Saúde. Coordenação de Informações Estratégicas. Boletim epidemiológico HIV - AIDS. Ano II n 1. Brasília DF: Editora MS; 2014.

6. BRASIL, M.S. Pacto Pela Saúde - Portaria 399/GM,22/02/2006. Dispõem o Pacto pela Saúde 2006 - Consolidação do SUS e aprova as Diretrizes Operacionais do Referido Pacto. Brasília (DF), 2006

7. Instituto Brasileiro de Geografia e Estatística (IBGE). Censo Demográfico 2010. [Internet] [acesso em Mar 2014]. Disponível em: http://cidades.ibge.gov.br/xtras/perfil.php?lang=\&codmun=431690\&search=||infogr\%E1ficos:-informa\%E7\%F5es-completas 
8. Reis CT, Czeresnia D, Barcellos Christovam, Tassinari WS. A interiorização da epidemia de HIVIAIDS e o fluxo intermunicipal de internação hospitalar na Zona da Mata, Minas Gerais, Brasil: uma análise espacial. Cad. Saúde Pública,jun 2008;24(6):1219-1228.

9. Szwarcwald CL, Bastos FI, Esteves MA, Andrade CLT. A disseminação da epidemia de AIDS no Brasil, no período de 1987-1996: uma análise espacial. Cad Saúde Pública 2000; 16 (1). p7-19.

10. Brito AM, Castilho EA, Szwarcwald CL. AIDS e infecção pelo HIV no Brasil: uma epidemia multifacetada. Ver Soc Bras MedTrop. 2000; 34: p 207-217.

11. Souza CC; Mata LRF; Azevedo C, Gomes CRG, Cruz GECP, Toffano SEM. Interiorização do HIVIAIDS no Brasil: um estudo epidemiológico. Rev. Bras. Ciên. Saúde, 2013; 11(35): p25-30.

12. Fonseca MGP, Szwarcwald CL, Bastos FI. Análise sociodemográfica da epidemia de AIDS no Brasil, 1987-1997. Ver. Saúde Publica. 2002; 36(6): p 678-685.

13. Santos NJS, Atrai A, Silva SR; Buçala CM, Laurenti R. aids no Estado de São Paulo. As mudanças no perfil da epidemia e perspectivas da vigilância epidemiológica. Rev. bras. epidemiol. 2002, 5(3): p 286-310.

14. Ministério da Saúde. Programa Nacional de DST e AIDS. Análise do banco de dados nacional de AIDS, 1980 a 2005, e gestante HIV+, 2000 a 2006. ed 3. Brasilia (DF): Editora MS. 2006.

15. Fonseca, MGP, Bastos FI. Twenty-five years of the Aids epidemic in Brazil: principal epidemiological findings, 19802005. Cad. de Saúde Pública. 2007; 23 (3): 533-544.

16.Lamptey PR, Johnson JL, Khan M. El desafio mundial del VIH y el SIDA. v61. Washingnton. PopulationBulletin, 2006. 17.Santos SMS, Oliveira MLF. (Com)vivendo com a Aids: perfil dos portadores de HIVIAIDS na região Noroeste do Estado do Paraná, 1989-2005. 2009; Maringá, 32 (1):51-56.

18. Taquette SR, Ruzany MH, Meirelles Z, Ricardo I.. Relacionamento violento na adolescência e risco de DST/Aids. Cad de Saúde Pública. 2003; 19: 1437-1444.

19. Lopes F. Para além das barreiras dos números: desigualdades raciais e saúde. Cad de Saúde Pública 2005; 21 (5): 1595-1601. 20. Associação Brasileira Multiprofissional de Proteção à Infância e à Adolescência. Abuso sexual de crianças e adolescentes. $3^{\mathrm{a}}$ Ed. Petrópolis (RJ): Autores \& Agentes \& Associados; 2002.

21. Taquette, SR. O paradoxo da moral sexual na adolescência e as DST/Aids. In: (Org.). Aids e juventude: gênero, classe e raça. Rio de Janeiro: EdUERJ, 2009. p. 135-154.

22. - PL Hottz, M Schechter A epidemiologia da infecção pelo HIV no Brasil e no mundo .2012, Cap 1: p 1-11. Ed. Permanyer Brasil Publicações, Ltda, disponível em: http://www.centrodegenomas.com.br/Arquivos/1/Livro_Infeccao_HIV. pdf\#page=14, acessado em 30 de setembro de 2014.

23. Brito AM, Castilho EA, Szwarcwald CL. AIDS e infecção pelo HIV no Brasil: uma epidemia multifacetada. Rev. Soc Bras Med Trop. 2001; 34(2): 207-217.

24. Rodrigues AL, Castilho EA. A epidemia de AIDS no Brasil, 1991-2000: descrição espaço temporal. Rev. Soc Bras Med Trop.2004;37(4):312-317.

25. Maschio, MBM, Balbino AP, De Souza PFR, Kalinke LP. Sexualidade na terceira idade: medidas de prevenção para doenças sexualmente transmissíveis e AIDS. Rev Gaúcha Enferm. 2011; 32(3):583-589.

26. Inelmen EM, Gasparini G, Enzi G. HIVIAIDS in older adults. A case report and literature review. Geriatrics. 2005;60:26-30.

27. Carvalho NS, Cho R, Flores LP. DST em populações indígenas no Brasil - Análise crítica e revisão da literatura. DST - J bras Doenças Sex Transm. 2011; 23(3):142-145.

28. Quermes PAA, Carvalho JA. O impacto dos benefícios assistenciais para os povos indígenas. Serv.Soc. Soc. 2013; (116): 769-791.

29. Cardoso MN, Caiaffa WT, MingotiAS.Projeto Ajude-Brasil II. AIDS incidence and mortality in injecting drug user: the Ajude-Brasil II Project. Cad. Saude Publica. 2006; 22(4):827-837.

30. GrangeiroA. Escuder MML, Castilho EA.Magnitude e tendência da epidemia de Aids em municípios brasileiros de 2002-2006.Rev.Saúde Publica. 2010, 44(3): 430-441.

31. Pechansky F, Diemen F, Inciardi JA, Surratt H, Boni R. Fatores de risco para transmissão do HIV em usuários de drogas de Porto Alegre, Rio Grande do Sul, Brasil Cad. Saúde Pública. 2004; 20(6): 1651-1660.

32. Parker R, Camargo Jr KR. Pobreza e HIVIAIDS: aspectos antropológicos e sociológicos. Cad. Saude Publica. 2000;16(1):89-102. 
33. Sampaio Neto LF, Novo NF, Silva SC, Condi, GG, PintoPCC. O impacto do conhecimento prévio da soropositividade em parturientes. Jornal Brasileiro de AIDS. 2003.4(2): $p$ 61-66.

34. Hagget $P$. The geographical structure of epidemics.Oxford.Oxford University Press; 2000.p10-24.

35. Tomazelli J, Czeresnia D, Barcellos C. Distribuição dos casos de AIDS em mulheres no Rio de Janeiro: uma análise espacial. Cad Saúde Pública 2004; 19:1049-1061.

36. Ministério da Saúde. Secretaria de Vigilância em Saúde. Programa Nacional de DST/AIDS. Contribuição dos centros de testagem e aconselhamento para universalizar o diagnóstico e garantir a eqüidade no acesso aos serviços. Brasília, DF: O Ministério; 2008.

\section{Édina Stadler Schossler}

Endereço para correspondência - Rua: Dr. Liberato Salzano Vieira da Cunha, $n^{\circ}$ 475, Ap 301, Bairro: Camobi, CEP: 97105-090, Santa Maria, RS, Brasil.

E-mail: edinaschossler@gmail.com

Lattes: http://lattes.cnpq.br/4513645201330029

Guilherme Adam Fraga - gadamfraga@hotmail.com

João Guilherme Stadler Schossler - joaogss_med@hotmail.com

Adalvane Nobres Damaceno - adalvane.damaceno@yahoo.com.br

Sandra Trevisan Beck - sbeck@ig.com.br

Teresinha Heck Weiller - Weiller2@hotmail.com

\section{Enviado em 08 de dezembro de 2014. Aceito em 07 de outubro de 2015.}

